\title{
On some properties of the Hoede-Bakker index*
}

\author{
AGNIESZKA RUSINOWSKA (a) \\ HARRIE DE SWART (b)
}

\begin{abstract}
(a) GATE, CNRS UMR 5824 - Université Lumière Lyon 2
93, Chemin des Mouilles - B.P.167, 69131 Ecully Cedex, France

E-mail: rusinowska@gate.cnrs.fr

Fax: + 33472866 090, Telephone: + 33472866080
\end{abstract}

(b) Tilburg University, Department of Philosophy, P.O. Box 90153

5000 LE Tilburg, The Netherlands

E-mail: H.C.M.deSwart@uvt.nl

Corresponding author: Agnieszka Rusinowska

*This paper has been published as Rusinowska and De Swart (2007) Journal of Mathematical Sociology 31(4): 267-293. 


\begin{abstract}
In this paper, we analyze the decisional power index, the so called Hoede-Bakker index. This index takes the preferences of the players into account, as well as the social structure in which players may influence each other. We investigate the properties of the Hoede-Bakker index. We adapt some postulates for power indices and voting power paradoxes for the Hoede-Bakker set up. We investigate whether this decisional power index displays the adapted voting power paradoxes and whether it satisfies the adapted postulates for power indices.
\end{abstract}

KEYWORDS: Hoede-Bakker index, power index, inclination vector, group decision, voting power paradoxes, postulates for power indices

RUNNING TITLE: On the Hoede-Bakker index 


\section{Introduction}

Many power indices have been introduced (Shapley and Shubik, 1954; Penrose, 1946; Banzhaf, 1965; Dubey and Shapley, 1979; Coleman, 1971; Johnston, 1978; Deegan and Packel, 1978; Holler, 1982; Holler and Packel, 1983; etc.). A review and a comparison of these power indices have been made, for instance, in Felsenthal and Machover (1998) or in Laruelle (2000). In the literature, some features of power indices, called voting power paradoxes, have been investigated, for instance, the paradox of new members and the paradox of large size (Brams, 1975; see also Brams and Affuso, 1976), the paradox of redistribution (Fischer and Schotter, 1978), and the quarrelling paradoxes (Kilgour, 1974). A theoretical analysis and extensive discussion of power indices and voting power paradoxes is given in Felsenthal and Machover (1998). There is also empirical research on paradoxes of power indices (see, for instance, Schotter, 1982). In particular, the occurrence of voting power paradoxes in real politics has been studied (Van Deemen and Rusinowska, 2003; Rusinowska, 2001; Rusinowska and Van Deemen, 2004). More recently, Felsenthal, Machover, and Zwicker (Felsenthal and Machover, 1995, 1998; Felsenthal, Machover, and Zwicker, 1998; see also Laruelle, 2000) proposed some desirable properties of power indices, which they refer to as postulates, the non-fulfilment of which they considered as paradoxical.

The aim of this paper is to investigate properties of the, as far as we know, less well-known Hoede-Bakker index. Hoede and Bakker (1982) introduced the concept of decisional power. The essential point of the Hoede-Bakker index is the distinction between the inclination to say 'yes' or 'no' and the final decision apparent in a vote. As Hoede and Bakker noticed, 'In fact, it is the difference between inclination and final decision in which the exertion of power on an actor manifests itself'. In our opinion, the Hoede-Bakker index is an interesting concept which deserves more attention, both from a theoretical and a practical point of view. First of all, the Hoede-Bakker index is an innovative concept in social network theories. It is defined for a social network, where influences among players are taken into account. It presents a natural way of determining the power of an actor in a network. The attractiveness of the Hoede-Bakker index is also related to the applicability of this index. The Hoede-Bakker index was already applied by Stokman and Willer (1999) to an analysis of Dutch and German coalitions.

In this paper, we adapt some postulates for power indices and voting power paradoxes (see, for instance, Felsenthal and Machover, 1998) to the special Hoede-Bakker set up. We propose some properties of the index defined for a social network, the non-fulfilment of which seems to be paradoxical. These properties are based on some parameters in a social network, like the number of players influenced by a given player and the number of players influencing a given player. A natural expectation may be that for a given network, the power of a player should be non-decreasing with respect to the first parameter mentioned and non-increasing with respect to the latter one. It seems also reasonable to assume that, for instance, by influencing a greater number of players and being 
influenced by a smaller number of players, a given player should become more powerful or, at least, should not lose power. Also forming a union in a social network should be rewarding to the players who decide to unite. It seems also reasonable to expect no actor to gain in terms of power from the appearance of a new actor if in the new situation all old members do not become more 'important' (i.e., they neither influence a greater number of players nor are influenced by a smaller number of players). The main aim of this paper is to check whether the Hoede-Bakker index has such desirable properties.

Our paper is organized as follows. Section 2 contains a short survey of postulates for power indices, and voting power paradoxes. All remaining sections, that is, Sections 3 - 8, concern the Hoede-Bakker index. In Section 3, we describe the decisional power index. In Section 4 , we show that this index depends on the way the group decision is made. Section 5 concerns the case with an even number of players. In Section 6, the decisional power index of a set of players is analyzed. In Section 7, we deal with some further properties of the Hoede-Bakker index. In particular, we check whether this index satisfies some postulates and displays some paradoxes. Finally, Section 8 contains conclusions. The paper also has an appendix. It contains figures of all social networks considered in the paper, tables with results for three figures, and a table (Table 4) summarizing the results.

\section{On Power Indices and their Properties}

A $(0,1)$-game is a pair $(N, v)$, where $N=\{1, \ldots, n\}$ denotes the set of players and $v: 2^{N} \rightarrow\{0,1\}$ is a function for which $v(\emptyset)=0$. A simple game is a $(0,1)$ game such that $v(N)=1$ and $v$ is nondecreasing, i.e., $v(S) \leq v(T)$ whenever $S \subseteq T \subset N$. Any nonempty subset of $N, \emptyset \neq S \subseteq N$ is called a coalition. A coalition $S$ is winning if $v(S)=1$, and losing if $v(S)=0$. Player $k \in S$ is a swinger in a winning coalition $S$, if his or her removal from this coalition makes it losing, that is, if $v(S)=1$ and $v(S \backslash\{k\})=0$. We refer to a winning coalition in which all players are swingers as a minimal winning coalition. If one player, say player $k \in N$, forms the only minimal winning coalition, then $k$ is called a dictator. A dummy is a player who is a member of no minimal winning coalition. Hence, a dictator, if there is one, can be characterized as the sole player who is not a dummy.

One interesting class of simple games is the class of weighted voting games. We use the symbol $\left[q ; w_{1}, w_{2}, \ldots, w_{n}\right]$ to represent a weighted voting game, where $q$ is the quota needed for a coalition to win, and $w_{k}$ is the number of votes of player $k(k=1,2, \ldots, n)$. The quota $q$ and the voting weights $w_{1}, w_{2}, \ldots, w_{n}$ are positive integers with $0<q \leq \sum_{k=1}^{n} w_{i}$. Then, the expression $\left[q ; w_{1}, w_{2}, \ldots, w_{n}\right]$ represents the simple game $v: 2^{N} \rightarrow\{0,1\}$ defined for all $S \subseteq N$ by $v(S)=1$ if $\sum_{k \in S} w_{k} \geq q$, and $v(S)=0$ otherwise.

A power index is a function $\phi$ that associates with each simple game $(N, v)$ a vector $\phi=\left(\phi_{1}, \ldots, \phi_{n}\right)$, where $\phi_{k}$ is interpreted as a measure of the influence that player $k$ can exert on the outcome. Many ideas on how to evaluate 
the distribution of power among the players have been proposed. One should mention, in particular, the Shapley-Shubik index, the normalized and absolute Banzhaf indices, the Coleman indices, the Deegan-Packel index, and the HollerPackel index. For a broad overview of some (well-known) power indices see, for instance, Felsenthal and Machover (1998) and Laruelle (2000).

Next, we recapitulate some postulates (also called axioms in the literature) concerning power indices and, closely related to the postulates, paradoxes of power indices (or, rather, properties that are not satisfied). The properties of voting power measures were presented in particular in Felsenthal and Machover (1998) and Laruelle (2000). We add a postulate concerning a dummy.

Invariance postulate - A voter's measure of power does not depend on his name.

Normalization postulate - The voters' measures of power add up to 1:

$$
\sum_{k \in N} \phi_{k}(v)=1
$$

Dummy postulate - A player $k$ is a dummy if and only if $\phi_{k}(v)=0$.

Monotonicity postulate - In a weighted voting body, a voter with a larger voting weight cannot be in a worse position than a voter with a smaller voting weight, i.e.,

$$
\text { if } w_{k}>w_{j} \text {, then } \phi_{k}(v) \geq \phi_{j}(v) \text {. }
$$

Bloc postulate - If two voters always vote together so that they end up forming a single voter, then the new voter has more power than each of the previous voters. Given $(N, v)$, we consider $\left(N \backslash\{j\}, v^{\prime}\right)$ such that $v^{\prime}(S)=v(S \cup\{j\})$ if $k \in S$, and $v^{\prime}(S)=v(S)$ otherwise. The bloc postulate requires that

$$
\text { if } \phi_{j}(v)>0 \text {, then } \phi_{k}\left(v^{\prime}\right)>\phi_{k}(v) \text {. }
$$

A violation of the bloc postulate leads essentially to the paradox of large size.

Paradox of large size (Brams, 1975) - This occurs when the power index of a union of parties is less than the sum of the power indices of the separate parties of that union. Let $P \subseteq N$ be the set of players who decided to unite. Let us call this union player $U$, where $w_{U}=\sum_{k \in P} w_{k}$. A power index $\phi$ displays the paradox of large size if

$$
\phi_{U}<\sum_{k \in P} \phi_{k}
$$

Donation postulate - In a weighted voting body, a voter cannot gain power by distributing some of his voting weight to other voters. Let $V=\left[q ; w_{1}, \ldots, w_{n}\right]$, $V^{\prime}=\left[q ; w_{1}^{\prime}, \ldots, w_{n}^{\prime}\right]$, and $\sum_{k=1}^{n} w_{k}=\sum_{k=1}^{n} w_{k}^{\prime}$. The donation postulate requires that

$$
\text { if } w_{j}^{\prime} \geq w_{j} \text { for each } j \neq k \text { and } w_{k}^{\prime}<w_{k} \text {, then } \phi_{k}\left(V^{\prime}\right) \leq \phi_{k}(V) \text {. }
$$


Assuming that more than one donor (i.e., a player getting a smaller weight) may exist, the paradox of redistribution expresses the violation of the donation postulate.

Paradox of redistribution (Fischer and Schotter, 1978) - This appears when either a party's voting weight decreases and at the same time its power index increases, or when a party gains in terms of voting weight, but loses in voting power. Let $V=\left[q ; w_{1}, \ldots, w_{n}\right], V^{\prime}=\left[q ; w_{1}^{\prime}, \ldots, w_{n}^{\prime}\right]$, and $\sum_{k=1}^{n} w_{k}=\sum_{k=1}^{n} w_{k}^{\prime}$. A power index $\phi$ displays the redistribution paradox if

$$
\text { for some } k, w_{k}^{\prime}<w_{k} \text { and } \phi_{k}\left(V^{\prime}\right)>\phi_{k}(V) \text {. }
$$

Paradox of new members (Brams, 1975; Brams and Affuso, 1976) - This appears when a new party joins the assembly and at least one old party has greater voting power in this new situation than in the old one. Let $V=\left[q ; w_{1}, \ldots, w_{n}\right]$ and $V^{\prime}=\left[q^{\prime} ; w_{1}, \ldots, w_{n}, w_{n+1}\right]$. A power index $\phi$ displays the paradox of new members if

$$
\text { for some } k \in N, \phi_{k}\left(V^{\prime}\right)>\phi_{k}(V) \text {. }
$$

\section{Description of the Hoede-Bakker Index}

In Brams and Affuso (1976), a distinction between an 'outcome oriented measure' and an 'actor-oriented measure' was discussed. The first measure is related to the ability of players to control outcomes. The latter definition focuses on the effects that actors have on other actors. The concept of decisional power was introduced by Hoede and Bakker (1982). The Hoede-Bakker index is an actor-oriented measure. Instead of measuring the power in terms of determining the outcome, this index is focused on the power of influencing players. The definition of decisional power (Hoede and Bakker, 1982) is based on the fact that, in situations in which decisions have to be made, the distinction between the inclination to say 'yes' or 'no' and the actual decision is taken into account. A player may, for instance, be in favor of a certain decision (a certain point at issue), that is, his inclination is then 'yes', but in fact he says 'no' due to the influence of other player(s).

In this section, we recapitulate the definition of the decisional power index (the Hoede-Bakker index). Let us consider the following situation. There are $n \geq 1$ players who have to make a decision about a certain point at issue (to accept or to reject a bill, a candidate, etc). By $N$ we denote the set of all players (actors, voters), that is, $N=\{1, \ldots, n\}$. With respect to the point at issue, each player has an inclination either to say 'yes' (denoted by ' 1 ') or 'no' (denoted by ' 0 '). Hence, for $n$ players, we have $2^{n}$ possible inclination vectors, that is, $n$-vectors consisting of zeros and ones. An inclination vector will be denoted by $i$, the set of all $n$-vectors by $I$. For $n=3$ we have 8 inclination vectors. For instance, $i=(1,1,0)$ means that player 1 and 2 have the inclination 'yes', but actor 3 has the inclination 'no'.

Due to the influence among actors, a player's decision may be different from his inclination. We then say that each inclination vector $i \in I$ is transformed 
into a decision vector, denoted by $b$. Formally, such a transformation may be represented by an operator, assigning to each inclination vector, a decision vector resulting from the influence among players. We denote this operator by $B: I \rightarrow I$, that is,

$$
b=B i .
$$

Decision vector $b$ is an $n$-vector consisting of zeros and ones $(b \in I)$ and indicating the decisions made by all actors. For instance, $B(1,1,0)=(1,0,0)$ means that players 1 and 3 voted according to their inclinations (player 1 said 'yes', player 3 - 'no'), and actor 2 decided for 'no', although his inclination was 'yes'. The set of all decision vectors is denoted by $B(I)$.

When all players made their individual decisions, a group decision must be taken. It is represented by an operator $g d$, assigning to each decision vector, a group decision (either 'yes' or 'no'). Formally, the group decision is then a function $g d: B(I) \rightarrow\{1,-1\}$ defined on the vectors $b$, and it has the value +1 if the group decision is 'yes' and the value -1 if the group decision is 'no'.

There are many possibilities to choose the operator $B$ and the group decision $g d$. Hoede and Bakker (1982) proposed two axioms which have to be satisfied by $B$ and $g d$. First, let us introduce some definitions.

Definition 3.1 For each inclination vector $i=\left(i_{1}, \ldots, i_{n}\right)$, the complement of $i$ is a vector $i^{c}=\left(i_{1}^{c}, \ldots, i_{n}^{c}\right)$ such that for each $k \in\{1, \ldots, n\}$

$$
i_{k}^{c}=\left\{\begin{array}{lll}
1 & \text { if } & i_{k}=0 \\
0 & \text { if } & i_{k}=1
\end{array} .\right.
$$

Definition 3.2

$$
i \leq i^{\prime} \Longleftrightarrow\left\{k \in N \mid i_{k}=1\right\} \subseteq\left\{k \in N \mid i_{k}^{\prime}=1\right\} .
$$

According to Hoede and Bakker (1982), $B$ and $g d$ have to satisfy the following two axioms:

AXIOM (A-1):

$$
\forall i \in I\left[g d\left(B i^{c}\right)=-g d(B i)\right]
$$

$\operatorname{AXIOM}(\mathrm{A}-2)$ :

$$
\forall i \in I \forall i^{\prime} \in I\left[i \leq i^{\prime} \Rightarrow g d(B i) \leq g d\left(B i^{\prime}\right)\right]
$$

Axiom (A-1) says that changing all inclinations leads to the opposite group decision. Axiom (A-2) means monotonicity, more precisely, the group decision 'yes' is not changed into 'no' if the set of players with inclination 'yes' is enlarged.

Assuming both axioms (A-1) and (A-2) to be satisfied, Hoede and Bakker defined a concept called the decisional power index or the Hoede-Bakker index. Taking all possible configurations of players' inclinations as equally probable, the Hoede-Bakker index of a player is equal to the difference between the proportion of times the group decision will follow that player's inclination, and the proportion of times, the group decision will be different from the inclination of the player in question. We have the following formal definition: 
Definition 3.3 Given B and gd, the decisional power index (the Hoede-Bakker index) of a player $k \in N$ is given by

$$
H B(k)=\frac{1}{2^{n-1}} \cdot \sum_{\left\{i: i_{k}=1\right\}} g d(B i) .
$$

Note that when defining this power index of a player, we assume this player to have an inclination 'yes' and consider the group decisions for all $2^{n-1}$ inclination vectors of the remaining players. If we assumed a player to have an inclination 'no' (and then considered the group decisions for all $2^{n-1}$ inclination vectors of the remaining players), by virtue of axiom (A-1), the Hoede-Bakker index would be a non-positive number with absolute value equal to that from Definition 3.3. Hence, without loss of generality, we assume the player has an inclination 'yes'.

Example 3.1 In order to demonstrate the usefulness of the Hoede-Bakker index, we consider a family network, consisting of three actors: player 1 (mother), player 2 (father), and player 3 (child). The mother is influenced by no family member, while the father happens to be influenced by his wife: he always decides according to the inclination of his wife. The child is clearly under influence of both parents. When their inclinations are the same, he follows the inclination of his parents, but when their inclinations differ from each other, the child feels free to follow his own inclination. Nevertheless, when making a family decision, the child's decision is never taken into account: the family will approve of a given proposal (i.e., the group decision will be 'yes') if and only if both parents decide for 'yes', otherwise the proposal is disapproved. This family network is illustrated by Figure 1(b), and the group decision is shown in Table 1 (see Appendix). What can we say about such a family situation? Which indices should we use?

First of all, let us notice that none of the 'standard' power indices considers a social network and, in particular, the influence between players. They all start from votes (i.e., decisions) of players. The Hoede-Bakker index is the only one we know of that takes into account the influence among players and what happens before the players make their decisions.

Let us apply then this index to our family network. The intuition says that the child is clearly powerless in this game: he follows his inclination only in the special case that his parents disagree, but he is never considered in the group decision. We expect that the situation of player 1 is the strongest one, but player 2 seems to have also some power, since the child respects him as much as player 1, and moreover, the father is involved in the group decision. The HoedeBakker index helps us to see the situation better. It happens that the index of the mother is equal to 1 , while both the indices of the father and the child are equal to 0 . We can say that the wife is a dictator (we will talk more about dictatorship later on), but actually we have to do with a kind of hidden dictator here. It is important to realize 'what is really going on' in a social network. The Hoede-Bakker index seems to be very appropriate for considering influence and power among players forming all kinds of networks: networks in families, 
institutions, politics, societies, etc. Hence, it is important to look carefully at properties of this index.

\section{Power Dependent on the Decision Operator}

Hoede and Bakker (1982) considered a social network modelled as a directed graph, and next, depending on the structure of the network, they defined the operator $B$ and the group decision operator $g d$. Note that, since $B$ and $g d$ have to satisfy only two axioms, (A-1) and (A-2), the definition of $B$ and $g d$ does not have to be unique. Because it will be used frequently below, we define a Standard Procedure. According to this procedure, a non-influenced player always follows his inclination, and an influenced player decides according to the inclination of the majority (i.e., more than half) of the actors influencing him. A group decision is made by simple majority. The formal definition is as follows:

Definition 4.1 Let $I N F(k)$ denote the set of players influencing player $k \in N$, $0 \leq|I N F(k)| \leq n-1, k \notin I N F(k), I N F^{y e s}(k, i)=\left\{m \in I N F(k) \mid i_{m}=1\right\}$, $I N F^{n o}(k, i)=\left\{m \in I N F(k) \mid i_{m}=0\right\}$, where $i=\left(i_{1}, \ldots, i_{n}\right)$ denotes an inclination vector. $B i=\left(b_{1}, \ldots, b_{n}\right)$ denotes the decision vector for $i$.

For $n$ odd, the Standard Procedure or Procedure 1 for the operator $B$ and the group decision operator gd is defined as follows. For each $k \in N$ :

1. If $|I N F(k)|=0$, then for each $i=\left(i_{1}, \ldots, i_{n}\right), b_{k}=i_{k}$.

2. If $|I N F(k)|>0$, then for each $i=\left(i_{1}, \ldots, i_{n}\right)$ :

(a) if $\left|I N F^{y e s}(k, i)\right|>\left|I N F^{n o}(k, i)\right|$, then $b_{k}=1$,

(b) if $\left|I N F^{\text {yes }}(k, i)\right|<\left|I N F^{\text {no }}(k, i)\right|$, then $b_{k}=0$,

(c) if $\left|I N F^{y e s}(k, i)\right|=\left|I N F^{n o}(k, i)\right|$, then $b_{k}=i_{k}$.

For each $i=\left(i_{1}, \ldots, i_{n}\right)$ :

$$
g d(B i)=\left\{\begin{array}{cc}
+1 & \text { if } \quad\left|\left\{k \in N \mid b_{k}=1\right\}\right| \geq\left[\frac{n}{2}\right]+1 \\
-1 & \text { otherwise }
\end{array}\right.
$$

where $[x]$ means the greatest integer not greater than $x$.

Proposition 4.1 For $n$ odd, the operators $B$ and gd, as defined in the Standard Procedure, satisfy both axioms (A-1) and (A-2).

Proof: Let $i=\left(i_{1}, \ldots, i_{n}\right)$ be arbitrary and let it contain $x 1$ 's and $n-x 0$ 's, $0 \leq x \leq n$. We determine $B i=\left(b_{1}, \ldots, b_{n}\right)$ and $g d(B i)$. Let $B i$ contain $y 1$ 's and $n-y 0$ 's. If $y \geq\left[\frac{n}{2}\right]+1$, then $g d(B i)=1$, otherwise $g d(B i)=-1$.

Now, let us consider $i^{c}$. It contains $n-x 1$ 's and $x 0$ 's. We determine $B i^{c}=$ $\left(b_{1}^{\prime}, \ldots, b_{n}^{\prime}\right)$. For each player $k$ such that $|I N F(k)|=0, b_{k}^{\prime}=i_{k}^{c}=-i_{k}=-b_{k}$. For each player $k$ such that $|I N F(k)|>0$, also $b_{k}^{\prime}=-b_{k}$. Then, $B i^{c}=$ $-\left(b_{1}, \ldots, b_{n}\right)=-B i$. Hence, $B i^{c}$ contains $y 0$ 's and $n-y 1$ 's. 
Suppose that $n$ is odd. Then, $\left[\frac{n}{2}\right]+1=\frac{n+1}{2}$. If $y \geq \frac{n+1}{2}$, then $g d(B i)=+1$ and $n-y \leq \frac{n-1}{2}<\frac{n+1}{2}$, which gives $g d\left(B i^{c}\right)=-1$. If $y<\frac{n+1}{2}$, then $g d(B i)=-1$ and $g d\left(B i^{c}\right)=+1$. If $n$ is even, then there is a problem when $y=n-y$. Then, $\left[\frac{n}{2}\right]=\frac{n}{2}, y=\frac{n}{2}<\frac{n}{2}+1$, and hence $g d(B i)=-1$. But then, also $n-y=\frac{n}{2}<\frac{n}{2}+1$ and $g d\left(B i^{c}\right)=-1$, which means that axiom (A-1) is NOT satisfied. However, axiom (A-2) is satisfied.

For a given $i=\left(i_{1}, \ldots, i_{n}\right)$ having $x 1^{\prime}$ 's and $n-x 0$ 's, we consider $i^{\prime}=\left(i_{1}^{\prime}, \ldots, i_{n}^{\prime}\right)$ such that $\left\{k \in N \mid i_{k}=1\right\} \subseteq\left\{k \in N \mid i_{k}^{\prime}=1\right\}$. Hence, in particular, $i^{\prime}$ has $x+\epsilon 1$ 's and $n-x-\epsilon 0$ 's, where $0 \leq \epsilon \leq n-x$. Let $B i=\left(b_{1}, \ldots, b_{n}\right)$ and $B i^{\prime}=\left(b_{1}^{\prime}, \ldots, b_{n}^{\prime}\right)$. Then, $\left|\left\{k \in N \mid b_{k}^{\prime}=1\right\}\right| \geq\left|\left\{k \in N \mid b_{k}=1\right\}\right|$. Hence, if $\left|\left\{k \in N \mid b_{k}=1\right\}\right| \geq\left[\frac{n}{2}\right]+1$, then $g d(B i)=+1$ and $g d\left(B i^{\prime}\right)=+1$. If $\left|\left\{k \in N \mid b_{k}=1\right\}\right|<\left[\frac{n}{2}\right]+1$, then $g d(B i)=-1$ and hence $g d\left(B i^{\prime}\right) \geq g d(B i)$.

For a social network with an arbitrary odd number of players, we can also apply a procedure slightly different from the Standard Procedure, which we will call Procedure 2. Under this procedure, an influenced player, when following a majority of his 'bosses', takes also his own inclination into account.

Definition 4.2 Let INF( $k)$ denote the set of players influencing player $k \in N$, $k \notin I N F(k), I N F 2^{\text {yes }}(k, i)=\left\{m \in I N F(k) \cup\{k\} \mid i_{m}=1\right\}$,

$I N F 2^{n o}(k, i)=\left\{m \in I N F(k) \cup\{k\} \mid i_{m}=0\right\}$, where $i=\left(i_{1}, \ldots, i_{n}\right)$ denotes the inclination vector. $B i=\left(b_{1}, \ldots, b_{n}\right)$ denotes the decision vector for $i$.

For $n$ odd, Procedure 2 for the operators $B$ and $g d$ is defined as follows. For each $k \in N$ :

1. If $|\operatorname{INF}(k)|=0$, then for each $i=\left(i_{1}, \ldots, i_{n}\right), b_{k}=i_{k}$.

2. If $|\operatorname{INF}(k)|>0$, then for each $i=\left(i_{1}, \ldots, i_{n}\right)$,

(a) if $\left|I N F 2^{\text {yes }}(k, i)\right|>\left|I N F 2^{\text {no }}(k, i)\right|$, then $b_{k}=1$,

(b) if $\left|I N F 2^{\text {yes }}(k, i)\right|<\left|I N F 2^{\text {no }}(k, i)\right|$, then $b_{k}=0$,

(c) if $\left|I N F 2^{\text {yes }}(k, i)\right|=\left|I N F 2^{\text {no }}(k, i)\right|$, then $b_{k}=i_{k}$.

For each $i=\left(i_{1}, \ldots, i_{n}\right)$,

$$
g d(B i)=\left\{\begin{array}{ccc}
+1 & \text { if } & \left|\left\{k \in N \mid b_{k}=1\right\}\right| \geq\left[\frac{n}{2}\right]+1 \\
-1 & \text { otherwise }
\end{array},\right.
$$

where $[x]$ means the greatest integer not greater than $x$.

Proposition 4.2 For $n$ odd, the operators $B$ and $g d$, as defined in Procedure 2, satisfy both axioms (A-1) and (A-2).

The proof of Proposition 4.2 is very similar to the proof of Proposition 4.1. The results of applying Procedures 1 and 2 can, of course, be different, as illustrated in Example 4.1. The power index depends on how we define $B$ and $g d$. The only requirement is that the axioms (A-1) and (A-2) have to be satisfied. 
Example 4.1 We apply the Standard Procedure and Procedure 2 to Figure 3(f) (see Appendix). Players 1, 3, 4, and 5 are not influenced, but player 2 is influenced by players 1,3 , and 4 . Tables 2 and 3 show the group decision for Figure 3(f) with the Standard Procedure and Procedure 2, respectively (see Appendix). The Standard Procedure yields the following results:

$H B(1)=H B(3)=H B(4)=\frac{1}{16} \cdot(12-4)=\frac{1}{2}$,

$H B(2)=H B(5)=\frac{1}{16} \cdot(8-8)=0$,

while when applying Procedure 2 , we get $H B(k)=\frac{1}{16} \cdot(11-5)=\frac{3}{8}$ for all $k$.

Note that Procedure 2, taking into account player 2's inclinations in some cases, appears to be fruitful not only for player 2 , but also for player 5 , who did not change his behavior. Players 1, 3, and 4 lost some of their power when applying Procedure 2.

\section{$5 \quad$ Even Number of Players}

For an even number of players, the operator $B$ and the group decision $g d$ described in the Standard Procedure satisfy axioms (A-1) and (A-2) if, for each inclination vector $i$, the decision vector $B i$ has a different number of 1 's and 0 's. So, if for an even number of players there is not a draw in $B i$, we can still apply the Standard Procedure.

Hoede and Bakker (1982) mainly considered an odd number of voters, but for $n$ even, they suggested to 'add an isolated dummy actor with prescribed voting behavior, e.g., 'yes' if that is the group decision in case of a draw'.

Example 5.1 We can apply our Standard Procedure to a two-player network, where player 1 influences player 2. In this particular case, (A-1) and (A-2) are satisfied. We get $H B(1)=1$, and $H B(2)=0$.

Unfortunately, we cannot apply the Standard Procedure to a two-player network, in which there are two independent players, because then axiom (A-1) would not be satisfied. We then add an isolated player, but we assume that he says 'no' in the case of a draw. We get the following result:

$g d(B(1,1))=\operatorname{gd}(1,1)=+1, \operatorname{gd}(B(1,0,0))=\operatorname{gd}(1,0,0)=-1$, $g d(B(0,1,0))=g d(0,1,0)=-1, g d(B(0,0))=g d(0,0)=-1$, and, finally, $H B(1)=H B(2)=0$.

\section{Decisional Power of a Set of Players}

Hoede and Bakker (1982) also defined the decisional power index of a set $S$ of players. In the definition, all actors in $S$ are assumed to have the same inclination 'yes', and we consider the group decisions for all $2^{n-|S|}$ inclination vectors of the others, where $|S|$ means, as usual, the number of players in $S$. 
Definition 6.1 Given B and gd, the decisional power index (the Hoede-Bakker index) of a set of players $S \subseteq N$ is given by

$$
H B(S)=\frac{1}{2^{n-|S|}} \cdot \sum_{\left\{i: i_{k}=1 \text { for all } k \in S\right\}} g d(B i) .
$$

Hoede and Bakker (1982) proved that for each set $S$ of players, $0 \leq H B(S) \leq 1$.

We would like to show some other properties of the decisional power of a set of players. It is straightforward to show that the power index of the whole set $N$ of players is maximal:

Fact 6.1 For each $N, H B(N)=1$.

Another property says that the larger the set of players, the greater (or equal) the Hoede-Bakker index.

Proposition 6.1 For each $S \subset S^{\prime}, H B(S) \leq H B\left(S^{\prime}\right)$.

Proof: Assuming $S \subset S^{\prime}$, there exists $S^{\prime \prime} \neq \emptyset$ such that $S^{\prime}=S \cup S^{\prime \prime}$ and $S \cap S^{\prime \prime}=\emptyset$. Hence, $\left|S^{\prime}\right|=|S|+\left|S^{\prime \prime}\right|$. Let us introduce the following notation:

$$
S^{+}=\left\{i \mid i_{k}=1 \text { for all } k \in S\right\} .
$$

Hence, of course,

$$
\left(S \cup S^{\prime \prime}\right)^{+}=\left\{i \mid i_{k}=1 \text { for all } k \in S \cup S^{\prime \prime}\right\} .
$$

Note that, for each $i=\left(i_{1}, \ldots, i_{n}\right) \in S^{+}$, there exists $i^{*} \in\left(S \cup S^{\prime \prime}\right)^{+}$(that is, $i_{k}^{*}=1$ for $\left.k \in S \cup S^{\prime \prime}\right)$ such that $i \leq i^{*}$ and $i_{m}^{*}=i_{m}$ for all $m \in N \backslash\left(S \cup S^{\prime \prime}\right)$. Hence, by virtue of axiom (A-2), $g d(B i) \leq g d\left(B i^{*}\right)$. In the formula for $H B(S)$, we will replace each $i \in S^{+}$by $i^{*} \in\left(S \cup S^{\prime \prime}\right)^{+}$having the same inclinations for each $m \in N \backslash\left(S \cup S^{\prime \prime}\right)$. Since $\left|S^{+}\right|=2^{\left|S^{\prime \prime}\right|} \cdot\left|\left(S \cup S^{\prime \prime}\right)^{+}\right|$, we have

$$
\begin{gathered}
H B(S)=\frac{1}{2^{n-|S|}} \cdot \sum_{\left\{i: i_{k}=1 \text { for all } k \in S\right\}} g d(B i) \leq \\
\frac{1}{2^{n-|S|}} \cdot \sum_{\left\{i: i_{k}=1 \text { for all } k \in S \cup S^{\prime \prime}\right\}} 2^{\left|S^{\prime \prime}\right|} \cdot g d(B i)= \\
\frac{2^{\left|S^{\prime \prime}\right|}}{2^{n-|S|}} \cdot \sum_{\left\{i: i_{k}=1 \text { for all } k \in S^{\prime}\right\}} g d(B i)=\frac{2^{\left|S^{\prime \prime}\right|}}{2^{n-|S|}} \cdot H B\left(S^{\prime}\right) \cdot 2^{n-\left|S^{\prime}\right|}=H B\left(S^{\prime}\right) .
\end{gathered}
$$

Remark 6.1 Note that the property described in Proposition 6.1 is a (weak) version of the BLOC POSTULATE (see Section 2) for the decisional power of a set of players. A larger set of players $S^{\prime}$, where $S \subset S^{\prime}$, has equal or more power than the set $S$ and, by analogy, than the set $S^{\prime} \backslash S$. As shown in Example 6.1 below, we can have $H B(S)=H B\left(S^{\prime}\right)$, even if $H B\left(S^{\prime} \backslash S\right)>0$. For this reason, we speak about a weak version of the bloc postulate. 
Conclusion 6.1 The Hoede-Bakker index of a set of players satisfies a weak version of the bloc postulate.

The last question we would like to discuss is the following:

$$
\text { Is there a relation between } H B(S) \text { and } \sum_{k \in S} H B(k) \text { ? }
$$

In fact, the answer is

\section{Anything may happen!}

Example 6.1 In order to show that each possible relation $(>,<$, and $=)$ between $H B(S)$ and $\sum_{k \in S} H B(k)$ may appear, we analyze Figure 3(e). When applying the Standard Procedure to this figure, we get

$H B(1)=\frac{5}{8}, H B(2)=H B(3)=\frac{3}{8}, H B(4)=H B(5)=\frac{1}{8}$.

By virtue of Definition 6.1, we get

$H B(\{1,5\})=\frac{1}{8} \cdot(7-1)=\frac{3}{4}=H B(1)+H B(5)$,

$H B(\{1,2,3\})=\frac{1}{4} \cdot(4-0)=1<\frac{11}{8}=H B(1)+H B(2)+H B(3)$,

$H B(\{2,3,4\})=\frac{1}{4} \cdot(4-0)=1>\frac{7}{8}=H B(2)+H B(3)+H B(4)$.

Note that $H B(N)=H B(\{2,3,4\})$, while $H B(\{1,5\})>0$ (see Remark 6.1).

In Example 6.1, we can recognize something like the PARADOX OF LARGE SIZE (see Section 2). The Hoede-Bakker index of a set of players may be less than the sum of the power indices of the players who constitute the set.

Conclusion 6.2 The decisional power index of a set of players may display the paradox of large size.

\section{Some Properties of the Hoede-Bakker Index}

In this section, we will analyze some further properties of the decisional power index of a player. In order to determine the properties of this index, sometimes we cannot apply the same postulates and paradoxes which were defined for voting games. Hence, we will first adapt some postulates and paradoxes mentioned in Section 2 for the Hoede-Bakker set-up. Next, we will check whether the adapted paradoxes occur for the Hoede-Bakker index. Moreover, we will check whether the Hoede-Bakker index satisfies the adapted postulates.

\subsection{Invariance and Normalization Postulates}

The invariance postulate is satisfied, that is, the Hoede-Bakker index of a player does not depend on his name. The normalization postulate is NOT satisfied by the Hoede-Bakker index. For instance, for Figure 3(f), with the Standard Procedure, we have $\sum_{k=1}^{5} H B(k)=\frac{3}{2}$. 
Definition 7.1 Given B and gd, a player $k \in N$ is called a dictator in a social network if the group decision is always the same as the inclination of player $k$, that is, if for each $i \in I$ such that $i_{k}=1, g d(B i)=+1$.

Note that from Axiom (A-1) and Definition 7.1 it follows that for each $i \in I$ such that $i_{k}=0, g d(B i)=-1$.

Hoede and Bakker (1982) defined a dictator as a player with a decisional power index equal to 1 . We introduced an equivalent definition of a dictator, but it is straightforward to show that:

Fact 7.1 Given B and gd for a social network,

$$
\forall k \in N[H B(k)=1 \Longleftrightarrow k \text { is a dictator }] .
$$

Note that a dictator does not have to influence all remaining players. We can show this by analyzing Figures $2(\mathrm{a})$ and $2(\mathrm{~b})$. In both cases, when applying the Standard Procedure, we have $H B(1)=1$ and $H B(k)=0$ for $k \in\{2,3,4\}$, which means that player 1 is a dictator. He has an influence on all remaining voters in the network presented in Figure 2(a), but he does not have an influence on player 4 in Figure 2(b).

Proposition 7.1 Given B and gd for a social network,

$$
\text { there is a dictator } \Rightarrow \sum_{k=1}^{n} H B(k)=1 \text {. }
$$

Proof: Suppose that there exists a dictator, say $k \in N$. Hence, for each $i \in I$, if $i_{k}=1$, then $g d(B i)=+1$, and if $i_{k}=0$, then $g d(B i)=-1$. Let us consider another arbitrary player $m \in N$. There are $2^{n-1}$ inclination vectors $i$ such that $i_{m}=1$ : among them, there are $2^{n-2}$ vectors with $i_{k}=1$ and $2^{n-2}$ with $i_{k}=0$. Moreover, for each $i$ such that $i_{m}=1$ and $i_{k}=1$, there is $i^{\prime}$ such that $i_{m}^{\prime}=1, i_{k}^{\prime}=0$ and $i_{j}^{\prime}=i_{j}$ for each $j \in N \backslash\{k, m\}$. Hence, $g d(B i)=+1$ and $g d\left(B i^{\prime}\right)=-1$, because $i_{k}=1, i_{k}^{\prime}=0$, and $k$ is a dictator. However, $\sum_{\left\{i: i_{m}=1\right\}} g d(B i)=2^{n-2}-2^{n-2}=0$, and, therefore, $\sum_{k=1}^{n} H B(k)=1$.

\subsection{Dummy Postulate}

One can easily show that

Fact 7.2 Given $B$ and $g d$,

$$
\begin{gathered}
\forall k \in N[H B(k)=0 \Longleftrightarrow \\
\left.\left|\left\{i \in I \mid i_{k}=1 \wedge g d(B i)=+1\right\}\right|=\left|\left\{i \in I \mid i_{k}=1 \wedge g d(B i)=-1\right\}\right|\right]
\end{gathered}
$$

In Section 2, we considered weighted voting games in which players have weights. When analyzing the Hoede-Bakker index, we will use different parameters, like the number of players influenced by a given voter, or the number of 
players influencing a given actor. For a given social network, and for player $k \in N$, we introduce the following notation:

$\alpha(k)$ - number of players influenced by player $k$

$$
\alpha(k)=|\{j \in N \mid k \in \operatorname{INF}(j)\}|
$$

$\gamma(k)$ - number of players influencing player $k$

$$
\gamma(k)=|I N F(k)|
$$

$\Lambda$ - number of influenced players in a social network

$$
\Lambda=|\{j \in N \mid \exists k \in N \backslash\{j\}[k \in I N F(j)]\}|
$$

$\Gamma$ - number of influencing players in a social network

$$
\Gamma=|\{j \in N \mid \exists k \in N \backslash\{j\}[j \in I N F(k)]\}| .
$$

One might ask whether there is a relation between the Hoede-Bakker index of a player $k$ being equal to 0 and the statement $\alpha(k)=0$ (there are no voters influenced by this player) or the statement $\gamma(k)>0$ (there are some actors influencing the given player). One can show that

Proposition 7.2 NONE of the following implications is true:

$$
\begin{aligned}
& \forall k \in N[H B(k)=0 \Rightarrow \alpha(k)=0] \\
& \forall k \in N[\alpha(k)=0 \Rightarrow H B(k)=0] \\
& \forall k \in N[H B(k)=0 \Rightarrow \gamma(k)>0] \\
& \forall k \in N[\gamma(k)>0 \Rightarrow H B(k)=0] .
\end{aligned}
$$

Proof: In order to show that the first and the third implications are not true, let us consider Figure $3(\mathrm{~g})$. Player 1 happens to be a dictator under the Standard Procedure: $H B(1)=1, H B(k)=0$ for $k \in\{2,3,4,5\}$. Hence, $H B(2)=0$, $\alpha(2)=1>0$, and $\gamma(2)=0$.

In order to prove that the second and the fourth implications are not true, we consider Figure 3(e). We have $\alpha(4)=0, \gamma(4)=2>0$, and $H B(4)=\frac{1}{8}>0$.

\subsection{Monotonicity}

It also appears that the number of players influencing a given voter is NOT proportional to the lack of power of the influenced player. One can prove that

Proposition 7.3 Given a social network, $B$ and $g d$, the Hoede-Bakker index does NOT have to be monotonic with respect to $\gamma$. It is NOT true that

$$
\forall k, k^{\prime} \in N\left[\left(\gamma(k)>\gamma\left(k^{\prime}\right) \wedge \alpha(k)=\alpha\left(k^{\prime}\right)\right) \Rightarrow H B(k) \leq H B\left(k^{\prime}\right)\right] .
$$


Proof: In order to show that $H B(k)$ is not monotonic with respect to $\gamma(k)$, we consider Figure 3(d). Under the Standard Procedure, we get $H B(1)=H B(2)=H B(4)=\frac{1}{2}, H B(3)=H B(5)=0$. Hence, $\gamma(4)=2>1=\gamma(3), \alpha(3)=\alpha(4)=0$, and $H B(4)=\frac{1}{2}>0=H B(3)$, and we showed that the implication mentioned is NOT true.

It also appears that the number of players influenced by a given voter does NOT have to illustrate the power of the influencing player if we apply, for instance, Procedure 2.

Proposition 7.4 Given a social network, B and gd, the Hoede-Bakker index does NOT have to be monotonic with respect to $\alpha$.

Proof: Let us apply Procedure 2 described in Definition 4.2 to Figure $3(\mathrm{j})$. We get $H B(1)=H B(2)=H B(4)=\frac{1}{4}$ and $H B(3)=H B(5)=\frac{1}{2}$. Hence, $\alpha(1)=2>1=\alpha(5), \gamma(1)=\gamma(5)=0$, but $H B(1)=\frac{1}{4}<\frac{1}{2}=H B(5)$.

If we consider TWO social networks with the same number of players, then the number of players influenced by a given voter does NOT have to illustrate the power of the influencing players. It is possible that a player who is influenced (and not influencing) in one social network has a greater power index in this network than in another network in which this player is influencing (but not influenced). When considering Figures 3(b) and 3(d), we prove that

Proposition 7.5 Given two social networks, $B$ and gd, the Hoede-Bakker index does NOT have to be monotonic with respect to $\alpha$. It is NOT true that

$$
\forall k \in N\left[\left(\alpha^{\prime}(k)>\alpha(k) \wedge \gamma^{\prime}(k) \leq \gamma(k)\right) \Rightarrow H B^{\prime}(k) \geq H B(k)\right],
$$

where $H B^{\prime}, \alpha^{\prime}$ and $\gamma^{\prime}$ refer to the second social network.

\subsection{Paradox of Redistribution and Donation Postulate}

In this subsection, we will check whether the redistribution paradox can appear for the Hoede-Bakker index, and whether the donation postulate (see Section 2) is satisfied for this index. In the redistribution paradox, we assume that $\sum_{k=1}^{n} w_{k}=\sum_{k=1}^{n} w_{k}^{\prime}$, where $w_{1}, \ldots, w_{n}$ and $w_{1}^{\prime}, \ldots, w_{n}^{\prime}$ denote the weights of the players in the two simple games considered. When looking for the redistribution paradox for the Hoede-Bakker index, we will assume that $\sum_{k=1}^{n} \alpha(k)=\sum_{k=1}^{n} \alpha^{\prime}(k)$, which is equivalent to $\sum_{k=1}^{n} \gamma(k)=\sum_{k=1}^{n} \gamma^{\prime}(k)$. In fact, $\sum_{k=1}^{n} \alpha(k)=\sum_{k=1}^{n} \gamma(k)$ and $\sum_{k=1}^{n} \alpha^{\prime}(k)=\sum_{k=1}^{n} \gamma^{\prime}(k)$ denote the number of 'arrows' in the first and the second social networks, respectively.

Definition 7.2 Let $\left((\alpha(k))_{k=1}^{n},(\gamma(k))_{k=1}^{n}, \Lambda, \Gamma\right),\left(\left(\alpha^{\prime}(k)\right)_{k=1}^{n},\left(\gamma^{\prime}(k)\right)_{k=1}^{n}, \Lambda^{\prime}, \Gamma^{\prime}\right)$ be the parameters of the two n-player social networks $A$ and $B$, respectively, where

$$
\sum_{k=1}^{n} \alpha(k)=\sum_{k=1}^{n} \alpha^{\prime}(k)
$$


The Hoede-Bakker index displays the paradox of redistribution if

for some $k, \alpha^{\prime}(k)<\alpha(k), \gamma^{\prime}(k)>\gamma(k)$, and $H B^{\prime}(k)>H B(k)$,

where $H B$ and $H B^{\prime}$ denote the Hoede-Bakker index in social networks $A$ and $B$, respectively.

Example 7.1 Let us compare Figures 3(c) and 3(d). In Figure 3(c), the Standard Procedure gives $H B(k)=\frac{3}{8}$ for each $k \in\{1,2,3,4,5\}$. Note that player 2 , influenced by all players, has the same power as the voters influencing him. Moreover, we have $\Lambda=1, \Gamma=4$, and, hence, $\Lambda+\Gamma=5, \alpha(4)=1, \gamma(4)=0$, $H B(4)=\frac{3}{8}$, and

$$
\sum_{k=1}^{5} \alpha(k)=\alpha(1)+\alpha(3)+\alpha(4)+\alpha(5)=4=\sum_{k=1}^{5} \gamma(k)=\gamma(2) .
$$

In Figure 3(d), we have $\Lambda^{\prime}=3, \Gamma^{\prime}=2$, hence additionally $\Lambda^{\prime}+\Gamma^{\prime}=5=\Lambda+\Gamma$,

$$
\sum_{k=1}^{5} \alpha^{\prime}(k)=\alpha^{\prime}(1)+\alpha^{\prime}(2)=4=\sum_{k=1}^{5} \alpha(k),
$$

and equivalently,

$$
\sum_{k=1}^{5} \gamma^{\prime}(k)=\gamma^{\prime}(3)+\gamma^{\prime}(4)+\gamma^{\prime}(5)=4=\sum_{k=1}^{5} \gamma(k),
$$

$\alpha^{\prime}(4)=0<1=\alpha(4), \gamma^{\prime}(4)=2>0=\gamma(4)$, but $H B^{\prime}(4)=\frac{1}{2}>\frac{3}{8}=H B(4)$.

By virtue of Example 7.1, we can conclude that

Conclusion 7.1 The Hoede-Bakker index may display the paradox of redistribution.

Let us redefine now the donation postulate for the Hoede-Bakker index.

Definition 7.3 Let $\left((\alpha(k))_{k=1}^{n},(\gamma(k))_{k=1}^{n}, \Lambda, \Gamma\right),\left(\left(\alpha^{\prime}(k)\right)_{k=1}^{n},\left(\gamma^{\prime}(k)\right)_{k=1}^{n}, \Lambda^{\prime}, \Gamma^{\prime}\right)$ be the parameters of the two n-player social networks $A$ and $B$, respectively, where

$$
\sum_{k=1}^{n} \alpha(k)=\sum_{k=1}^{n} \alpha^{\prime}(k)
$$

The donation postulate for the Hoede-Bakker index requires that

$$
\begin{gathered}
\text { if } \alpha^{\prime}(j) \geq \alpha(j) \text { and } \gamma^{\prime}(j) \leq \gamma(j) \text { for each } j \neq k, \text { and } \\
\alpha^{\prime}(k)<\alpha(k) \text { and } \gamma^{\prime}(k)>\gamma(k) \text {, then } H B^{\prime}(k) \leq H B(k),
\end{gathered}
$$

where $H B$ and $H B^{\prime}$ denote the Hoede-Bakker index in social networks $A$ and $B$, respectively. 
Example 7.2 In order to show that the donation postulate described in Definition 7.3 is not satisfied by the Hoede-Bakker index, we compare Figures 3(i) and 3(d). In Figure 3(i), under the Standard Procedure we have $H B(1)=1$, $H B(k)=0$ for each $k \in\{2,3,4,5\}$, and $\alpha(1)=2, \alpha(2)=1, \alpha(3)=0, \alpha(4)=1$, $\alpha(5)=0, \gamma(1)=0, \gamma(2)=1, \gamma(3)=1, \gamma(4)=1, \gamma(5)=1$, and $H B(4)=0$.

In Figure 3(d), we have $\alpha^{\prime}(1)=2, \alpha^{\prime}(2)=2, \alpha^{\prime}(3)=0, \alpha^{\prime}(4)=0, \alpha^{\prime}(5)=0$, and $\gamma^{\prime}(1)=0, \gamma^{\prime}(2)=0, \gamma^{\prime}(3)=1, \gamma^{\prime}(4)=2, \gamma^{\prime}(5)=1$, and $H B^{\prime}(4)=\frac{1}{2}$.

Hence, we get

$$
\sum_{k=1}^{5} \alpha(k)=\sum_{k=1}^{5} \alpha^{\prime}(k)=4
$$

and, of course,

$$
\begin{gathered}
\sum_{k=1}^{5} \gamma(k)=\sum_{k=1}^{5} \gamma^{\prime}(k)=4, \\
\forall j \neq 4\left[\alpha^{\prime}(j) \geq \alpha(j) \wedge \gamma^{\prime}(j) \leq \gamma(j)\right],
\end{gathered}
$$

and, moreover, $\alpha^{\prime}(4)<\alpha(4), \gamma^{\prime}(4)>\gamma(4)$, and $H B^{\prime}(4)>H B(4)$.

Hence, by virtue of Example 7.2, we can conclude that

Conclusion 7.2 The Hoede-Bakker index does not satisfy the donation postulate.

\subsection{Paradox of Large Size and Bloc Postulate}

As was shown in Section 6 (see Conclusion 6.2), the decisional power index of a set of players may display the paradox of large size. Now, we will check whether the Hoede-Bakker index of an individual player displays this paradox as well. We assume that if some voters create an union, then the new player-union will influence all voters who were influenced by the players forming the union, and moreover, the voter-union will be influenced by all voters who influenced at least one member of the union. Since a player cannot influence himself, if a voter influenced by another one forms a union with his 'boss', this influence will be, in a sense, lost. We can redefine the paradox of large size for the Hoede-Bakker index of an individual player in the following way:

Definition 7.4 Let $\left((\alpha(k))_{k=1}^{n},(\gamma(k))_{k=1}^{n}, \Lambda, \Gamma\right)$ be the parameters of $n$-player social network $A$. Let $P \subseteq N$ be the set of players who form a single playerunion $U$, resulting in a new social network $B$ with $N \backslash P \cup\{U\}$ as the set of players. We assume that

$$
\begin{aligned}
& \forall k \in P \forall j \in N \backslash P[k \in I N F(j) \Rightarrow U \in I N F(j)], \\
& \forall k \in P \forall j \in N \backslash P[j \in I N F(k) \Rightarrow j \in I N F(U)] .
\end{aligned}
$$

The Hoede-Bakker index displays the paradox of large size if

$$
H B^{\prime}(U)<\sum_{k \in P} H B(k),
$$


where $H B$ and $H B^{\prime}$ denote the Hoede-Bakker index in social networks $A$ and $B$, respectively.

In this subsection, we will also check whether the bloc postulate, the stronger property of a power index, is satisfied by the Hoede-Bakker index of a separate player. According to this postulate, if two players form a single voter, then the new voter has more power than each of the previous players. As was shown in Section 6 (see Conclusion 6.1), a weak version of the bloc postulate is satisfied by the Hoede-Bakker index of a set of players. We introduce the following definition of the bloc postulate for the Hoede-Bakker index of an individual player:

Definition 7.5 Let $\left((\alpha(k))_{k=1}^{n},(\gamma(k))_{k=1}^{n}, \Lambda, \Gamma\right)$ be the parameters of $n$-player social network $A$. Let $P \subseteq N$ be the set of players who form a single playerunion $U$, resulting in a new social network $B$ with $N \backslash P \cup\{U\}$ as the set of players. We assume that

$$
\begin{gathered}
\forall k \in P \forall j \in N \backslash P[k \in I N F(j) \Rightarrow U \in I N F(j)], \\
\forall k \in P \forall j \in N \backslash P[j \in I N F(k) \Rightarrow j \in I N F(U)], \\
\forall k \in P[H B(k)>0] .
\end{gathered}
$$

The bloc postulate for the Hoede-Bakker index requires that

$$
\forall k \in P\left[H B^{\prime}(U)>H B(k)\right],
$$

where $H B$ and $H B^{\prime}$ mean the Hoede-Bakker index in social networks $A$ and $B$, respectively.

Example 7.3 Let us consider Figure 3(e). The Hoede-Bakker indices are as follows: $H B(1)=\frac{5}{8}, H B(2)=H B(3)=\frac{3}{8}$, and $H B(4)=H B(5)=\frac{1}{8}$.

Let us suppose now that players 1,2 , and 3 decide to unite, resulting in only three players: player $1+2+3$, player 4 , and player 5 . Since in Figure $3(\mathrm{e})$, player 4 is influenced by voters 1 and 2 , and player 5 is influenced by voters 1 and 3 , the new player $1+2+3$ will influence actors 4 and 5 . Hence, we get Figure $1(\mathrm{c})$ with $1+2+3$ instead of 1,4 instead of 2 , and 5 instead of 3 . Hence, $H B^{\prime}(1+2+3)=1, H B^{\prime}(4)=H B^{\prime}(5)=0$.

We can recognize an occurrence of the paradox of large size, because $H B^{\prime}(1+2+3)=1<\frac{11}{8}=H B(1)+H B(2)+H B(3)$.

We start again with Figure 3(e), but now we assume that players 2, 3, and 4 decide to form one player $2+3+4$. In this case, player 2 loses his influence on player 4 , but player 4 should gain, because there is only one voter influencing him (that is, player 1) and moreover, owing to his union with player 3 , he will influence voter 5 . The new situation is described by Figure 1 (b) with $2+3+4$ instead of 2 and 5 instead of 3 . Hence, we get $H B^{\prime \prime}(1)=\frac{1}{4} \cdot(4-0)=1, H B^{\prime \prime}(2+3+4)=H B^{\prime \prime}(5)=\frac{1}{4} \cdot(2-2)=0$, which means that the bloc postulate is not satisfied and that all players forming the new voter $2+3+4$ lose power, even player 4 :

$H B^{\prime \prime}(2+3+4)=0<\frac{3}{8}=H B(2), H B^{\prime \prime}(2+3+4)=0<\frac{3}{8}=H B(3)$, and $H B^{\prime \prime}(2+3+4)=0<\frac{1}{8}=H B(4)$. 
By virtue of Example 7.3, we can conclude that

Conclusion 7.3 The Hoede-Bakker index may display the paradox of large size.

Conclusion 7.4 The Hoede-Bakker index does not satisfy the bloc postulate.

Of course, Conclusion 7.3 follows from Conclusion 7.4, because if the bloc postulate is not satisfied, then the new single voter-union has smaller (or equal) power than at least one previous voter in the union. Hence, the index of the voter-union is smaller than the sum of the indices of the players forming the union.

\subsection{Paradox of New Members}

In this subsection, we will show that the Hoede-Bakker index may display the paradox of new members. Let us first redefine the paradox of new members for the Hoede-Bakker index.

Definition 7.6 Let $\left((\alpha(k))_{k=1}^{n},(\gamma(k))_{k=1}^{n}, \Lambda, \Gamma\right),\left(\left(\alpha^{\prime}(k)\right)_{k=1}^{n+n^{\prime}},\left(\gamma^{\prime}(k)\right)_{k=1}^{n+n^{\prime}}, \Lambda^{\prime}, \Gamma^{\prime}\right)$ be the parameters of two n-player social networks $A$ and $B$, respectively, where $B$ is obtained from $A$ by adding $n^{\prime} \geq 1$ players in such a way that

$$
\forall_{k=1}^{n}\left[\alpha^{\prime}(k) \leq \alpha(k) \wedge \gamma^{\prime}(k) \geq \gamma(k)\right]
$$

The Hoede-Bakker index displays the paradox of new members if

$$
\text { for some } k \in\{1, \ldots, n\}, H B^{\prime}(k)>H B(k),
$$

where $H B$ and $H B^{\prime}$ denote the Hoede-Bakker index in social networks $A$ and $B$, respectively.

Example 7.4 We consider two examples (both starting with Figure 1(a)) in which the paradox of new members occurs. Since the Hoede-Bakker index satisfies the invariance postulate, we may change the name of player 3 in Figure 1 (a). Let us call him player 5. Hence, we have $\alpha(1)=1, \gamma(1)=0, \alpha(2)=0$, $\gamma(2)=1, \alpha(5)=0, \gamma(5)=0, H B(1)=1$ and $H B(2)=H B(5)=0$.

Let us suppose that two new players appear: player 3 influencing voter 2 and player 4 as an independent player. Hence, we get Figure $3(\mathrm{~h})$ :

$\alpha^{\prime}(1)=1, \gamma^{\prime}(1)=0, \alpha^{\prime}(2)=0, \gamma^{\prime}(2)=2, \alpha^{\prime}(5)=0, \gamma^{\prime}(5)=0$, $H B^{\prime}(1)=H B^{\prime}(3)=\frac{1}{2}$ and $H B^{\prime}(2)=H B^{\prime}(4)=H B^{\prime}(5)=\frac{1}{4}$.

This means that two old players, voter 2 and voter 5 , gained in power after players 3 and 4 entered, because $H B^{\prime}(k)=\frac{1}{4}>0=H B(k)$ for $k \in\{2,5\}$.

If for Figure 1(a) with players 1, 2, and 5, we add two independent players (voter 3 and voter 4 ), then we get Figure $3(\mathrm{~b})$. We then have

$\alpha^{\prime \prime}(1)=1, \gamma^{\prime \prime}(1)=0, \alpha^{\prime \prime}(2)=0, \gamma^{\prime \prime}(2)=1, \alpha^{\prime \prime}(5)=0, \gamma^{\prime \prime}(5)=0$, $H B^{\prime \prime}(1)=\frac{3}{4}, H B^{\prime \prime}(2)=0$ and $H B^{\prime \prime}(k)=\frac{1}{4}$ for $k \in\{3,4,5\}$,

which means that we face again the paradox of new members, because $H B^{\prime \prime}(5)=\frac{1}{4}>0=H B(5)$.

Conclusion 7.5 The Hoede-Bakker index may display the paradox of new members. 


\section{Conclusions}

In this paper, we analyzed the decisional power index (the Hoede-Bakker index). Given a social network, different operators $B$ and group decision operators $g d$ can be constructed that satisfy the two axioms of Hoede and Bakker for $B$ and $g d$, but may yield different outcomes. This confirms that the decisional power of an individual is dependent on the operators $B$ and $g d$. We proposed a Standard Procedure for $B$ and $g d$ which satisfies all adopted axioms for a network with an arbitrary odd number of players. Also, we discussed the case of an even number of players and we considered the decisional power index of a set of players.

We re-defined some postulates for power indices and a number of voting power paradoxes, adapting them to a social network and the Hoede-Bakker set up. Our postulates and paradoxes defined for the Hoede-Bakker index are dependent on some parameters of a social network, and they are not equivalent to the postulates and paradoxes defined for voting games. Using examples, we showed that the Hoede-Bakker index displays our re-defined paradoxes: the paradox of redistribution, the paradox of large size, and the paradox of new members. In addition, we showed that the Hoede-Bakker index does not satisfy some of our re-defined postulates: the monotonicity, the donation, and the bloc postulate.

The aim of the paper was to analyze the original Hoede-Bakker index defined by Hoede and Bakker (1982). The paper was concentrated on the properties of the Hoede-Bakker index, as stated in the title. In particular, we did not consider any possible 'improvements' and any applications of this index. There are several possible directions for further research on the Hoede-Bakker index. One may analyze the theoretical value of this index in a more detailed way, including some possible extensions of the index. Another direction concerns applications of the Hoede-Bakker index. As mentioned in the Introduction, the Hoede-Bakker index has already been applied to politics, in particular, to Dutch parliament. In our future research, we intend to work on further applications of this index. In particular, we would like to apply the Hoede-Bakker index to some European parliaments. Stokman and Willer (1999) applied the HoedeBakker index, in particular, to Dutch parties, using party orderings given by van Roozendaal $(1992,1993)$. We would like to apply the Hoede-Bakker index, in particular, to Polish parliament. In the Polish literature on Political Science there are results concerning party orderings. Based on some questionnaire experiments among Polish parties, Haman (2001) analyzed an ideological dimension for Polish parties. His results were applied, in particular, by Jasinski (2002) to construct social networks concerning Polish parties. Based on the results mentioned above, there are clearly many possibilities for applying the Hoede-Bakker index. 


\section{References}

[1] Banzhaf, J.: (1965), Weighted voting doesn't work: A mathematical analysis, Rutgers Law Review 19: 317-343.

[2] Brams, S.J.: (1975), Game Theory and Politics. New York, Free Press.

[3] Brams, S.J. and Affuso, P.: (1976), Power and size: A new paradox, Theory and Decision 7: 29-56.

[4] Deegan, J. and Packel, E.W.: (1978), A new index of power for simple n-person games, International Journal of Game Theory 7: 113-123.

[5] Dubey, P. and Shapley, L.S.: (1979), Mathematical properties of the Banzhaf power index, Mathematics of Operations Research 4: 99-131.

[6] Felsenthal, D.S. and Machover, M.: (1995), Postulates and paradoxes of relative voting power - a critical reappraisal, Theory and Decision 38: 195229 .

[7] Felsenthal, D.S. and Machover, M.: (1998), The Measurement of Voting Power: Theory and Practice, Problems and Paradoxes. London: Edward Elgar Publishers.

[8] Felsenthal, D.S., Machover, M. and Zwicker, W.S.: (1998), The bicameral postulates and indices of a priori voting power, Theory and Decision 44: 83-116.

[9] Fischer, D. and Schotter, A.: (1978), The inevitability of the paradox of redistribution in the allocation of voting weights, Public Choice 33: 49-67.

[10] Haman, J.: (2001), Is there left and right in parliament?, in: W. Wesolowski (ed.) Responsible for Politics (in Polish), 61-76. Warsaw: IFiS PAN.

[11] Hoede, C. and Bakker, R.: (1982), A theory of decisional power, Journal of Mathematical Sociology 8: 309-322.

[12] Holler, M.J.: (1982), Forming coalitions and measuring voting power, Political Studies 30: 262-271.

[13] Holler, M.J. and Packel, E.W.: (1983), Power, luck and the right index, Journal of Economics 43 (1983) 21-29.

[14] Jasinski, M.: (2002), Power Indices of Participants of Political Games, Ph.D. thesis (in Polish), Warsaw: Warsaw University, Institute of Sociology.

[15] Kilgour, D.M.: (1974), A Shapley value for cooperative games with quarreling, in: A. Rapoport (ed.) Game Theory as a Theory of Conflict Resolution, 193-206. Boston: Reidel.

[16] Laruelle, A.: (2000), On the choice of a power index, Universidad de Alicante, Alicante, Spain. 
[17] Penrose, L.S.: (1946), The elementary statistics of majority voting, Journal of the Royal Statistical Society 109: 53-57.

[18] Rusinowska, A.: (2001), Paradox of redistribution in Polish politics, The Annals of the Marie Curie Fellowships (MCFA), volume II, pp. 46-54. Brussels.

[19] Rusinowska, A. and Van Deemen, A.: (2005), The redistribution paradox and the paradox of new members in the German parliament, in: L.A. Petrosjan and V.V. Mazalov (eds.) Game Theory and Applications. Volume X, pp. 153-174. Nova Science Publishers. Inc. New York.

[20] Schotter, A.: (1982), The paradox of redistribution: some theoretical and empirical results, in: Holler, M. J. (ed.) Power, Voting, and Voting Power. Wurzburg-Wien: Physica-Verlag.

[21] Shapley, L.S.: (1953), A value for n-person games, Annals of Mathematics Studies 28: 307-317.

[22] Shapley, L.S. and Shubik, M.: (1954), A method for evaluating the distribution of power in a committee system, American Political Science Review 48: 787-792.

[23] Stokman, F. and Willer, D.: (1999), Exchange networks, power indices, and cabinet formation, unpublished paper.

[24] Van Deemen, A.M.A. and Rusinowska, A.: (2003), Paradoxes of voting power in Dutch politics, Public Choice 115: 109-137.

[25] Van Roozendaal, P.: (1992), Cabinets in Multi-Party Democracies. Amsterdam: Thesis Publishers.

[26] Van Roozendaal, P.: (1993), Cabinets in the Netherlands (1918-1990): The importance of 'dominant' and 'central' parties, European Journal of Political Research 23: 35-54. 


\section{Appendix}

Figure 1: Examples of social network for $n=3$

(a)

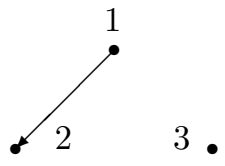

(b)

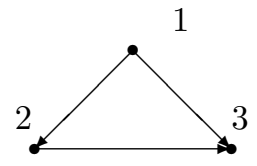

(c)

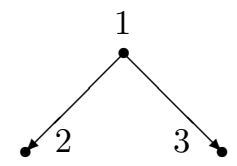

Figure 2: Examples of social network for $n=4$

(a)

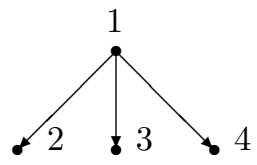

(b)

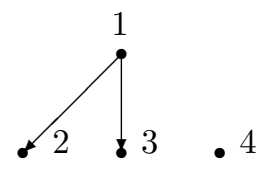

Figure 3: Examples of social network for $n=5$

(a) $\quad \begin{array}{lllll}1 & 2 & 3 & 4 & 5 \\ \bullet & \bullet & \bullet & \bullet & \bullet\end{array}$

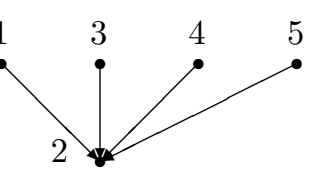

(e)

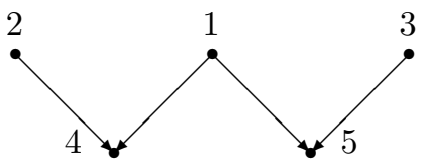

(g)

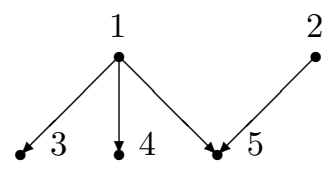

(i)

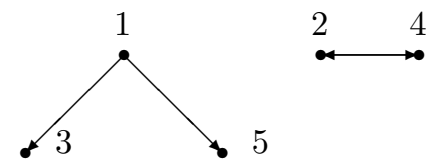

(b) $\quad \begin{array}{llll}1 & 3 & 4 & 5 \\ \bullet & \bullet & \bullet & \bullet\end{array}$

(d)

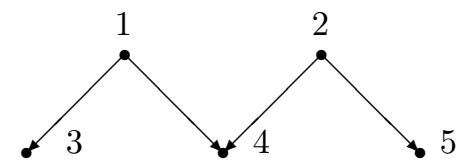

(f)

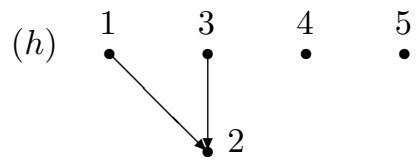

(j)

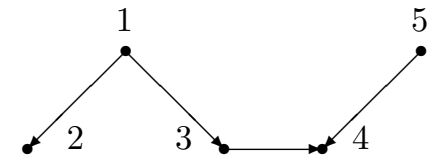


Table 1: Group decision for Figure 1(b) - family network

\begin{tabular}{|c|c|c|c|c|c|} 
inclination $i$ & $B i$ & $g d(B i)$ & inclination $i$ & $B i$ & $g d(B i)$ \\
\hline$(1,1,1)$ & $(1,1,1)$ & +1 & $(0,0,0)$ & $(0,0,0)$ & -1 \\
\hline$(1,1,0)$ & $(1,1,1)$ & +1 & $(0,0,1)$ & $(0,0,0)$ & -1 \\
\hline$(1,0,1)$ & $(1,1,1)$ & +1 & $(0,1,0)$ & $(0,0,0)$ & -1 \\
\hline$(0,1,1)$ & $(0,0,1)$ & -1 & $(1,0,0)$ & $(1,1,0)$ & +1 \\
\hline
\end{tabular}

Table 2: Group decision for Figure 3(f) with Standard Procedure

\begin{tabular}{|c|c|c|c|c|c|} 
inclination $i$ & $B i$ & $g d(B i)$ & inclination $i$ & $B i$ & $g d(B i)$ \\
\hline$(1,1,1,1,1)$ & $(1,1,1,1,1)$ & +1 & $(0,0,0,0,0)$ & $(0,0,0,0,0)$ & -1 \\
\hline$(1,1,1,1,0)$ & $(1,1,1,1,0)$ & +1 & $(0,0,0,0,1)$ & $(0,0,0,0,1)$ & -1 \\
\hline$(1,1,1,0,1)$ & $(1,1,1,0,1)$ & +1 & $(0,0,0,1,0)$ & $(0,0,0,1,0)$ & -1 \\
\hline$(1,1,0,1,1)$ & $(1,1,0,1,1)$ & +1 & $(0,0,1,0,0)$ & $(0,0,1,0,0)$ & -1 \\
\hline$(1,0,1,1,1)$ & $(1,1,1,1,1)$ & +1 & $(0,1,0,0,0)$ & $(0,0,0,0,0)$ & -1 \\
\hline$(0,1,1,1,1)$ & $(0,1,1,1,1)$ & +1 & $(1,0,0,0,0)$ & $(1,0,0,0,0)$ & -1 \\
\hline$(1,1,1,0,0)$ & $(1,1,1,0,0)$ & +1 & $(0,0,0,1,1)$ & $(0,0,0,1,1)$ & -1 \\
\hline$(1,1,0,1,0)$ & $(1,1,0,1,0)$ & +1 & $(0,0,1,0,1)$ & $(0,0,1,0,1)$ & -1 \\
\hline$(1,0,1,1,0)$ & $(1,1,1,1,0)$ & +1 & $(0,1,0,0,1)$ & $(0,0,0,0,1)$ & -1 \\
\hline$(0,1,1,1,0)$ & $(0,1,1,1,0)$ & +1 & $(1,0,0,0,1)$ & $(1,0,0,0,1)$ & -1 \\
\hline$(1,1,0,0,1)$ & $(1,0,0,0,1)$ & -1 & $(0,0,1,1,0)$ & $(0,1,1,1,0)$ & +1 \\
\hline$(1,0,1,0,1)$ & $(1,1,1,0,1)$ & +1 & $(0,1,0,1,0)$ & $(0,0,0,1,0)$ & -1 \\
\hline$(0,1,1,0,1)$ & $(0,0,1,0,1)$ & -1 & $(1,0,0,1,0)$ & $(1,1,0,1,0)$ & +1 \\
\hline$(1,0,0,1,1)$ & $(1,1,0,1,1)$ & +1 & $(0,1,1,0,0)$ & $(0,0,1,0,0)$ & -1 \\
\hline$(0,1,0,1,1)$ & $(0,0,0,1,1)$ & -1 & $(1,0,1,0,0)$ & $(1,1,1,0,0)$ & +1 \\
\hline$(0,0,1,1,1)$ & $(0,1,1,1,1)$ & +1 & $(1,1,0,0,0)$ & $(1,0,0,0,0)$ & -1 \\
\hline
\end{tabular}


Table 3: Group decision for Figure 3(f) with Procedure 2

\begin{tabular}{|c|c|c|c|c|c|} 
inclination $i$ & $B i$ & $g d(B i)$ & inclination $i$ & $B i$ & $g d(B i)$ \\
\hline$(1,1,1,1,1)$ & $(1,1,1,1,1)$ & +1 & $(0,0,0,0,0)$ & $(0,0,0,0,0)$ & -1 \\
\hline$(1,1,1,1,0)$ & $(1,1,1,1,0)$ & +1 & $(0,0,0,0,1)$ & $(0,0,0,0,1)$ & -1 \\
\hline$(1,1,1,0,1)$ & $(1,1,1,0,1)$ & +1 & $(0,0,0,1,0)$ & $(0,0,0,1,0)$ & -1 \\
\hline$(1,1,0,1,1)$ & $(1,1,0,1,1)$ & +1 & $(0,0,1,0,0)$ & $(0,0,1,0,0)$ & -1 \\
\hline$(1,0,1,1,1)$ & $(1,1,1,1,1)$ & +1 & $(0,1,0,0,0)$ & $(0,0,0,0,0)$ & -1 \\
\hline$(0,1,1,1,1)$ & $(0,1,1,1,1)$ & +1 & $(1,0,0,0,0)$ & $(1,0,0,0,0)$ & -1 \\
\hline$(1,1,1,0,0)$ & $(1,1,1,0,0)$ & +1 & $(0,0,0,1,1)$ & $(0,0,0,1,1)$ & -1 \\
\hline$(1,1,0,1,0)$ & $(1,1,0,1,0)$ & +1 & $(0,0,1,0,1)$ & $(0,0,1,0,1)$ & -1 \\
\hline$(1,0,1,1,0)$ & $(1,1,1,1,0)$ & +1 & $(0,1,0,0,1)$ & $(0,0,0,0,1)$ & -1 \\
\hline$(0,1,1,1,0)$ & $(0,1,1,1,0)$ & +1 & $(1,0,0,0,1)$ & $(1,0,0,0,1)$ & -1 \\
\hline$(1,1,0,0,1)$ & $(1,1,0,0,1)$ & +1 & $(0,0,1,1,0)$ & $(0,0,1,1,0)$ & -1 \\
\hline$(1,0,1,0,1)$ & $(1,0,1,0,1)$ & +1 & $(0,1,0,1,0)$ & $(0,1,0,1,0)$ & -1 \\
\hline$(0,1,1,0,1)$ & $(0,1,1,0,1)$ & +1 & $(1,0,0,1,0)$ & $(1,0,0,1,0)$ & -1 \\
\hline$(1,0,0,1,1)$ & $(1,0,0,1,1)$ & +1 & $(0,1,1,0,0)$ & $(0,1,1,0,0)$ & -1 \\
\hline$(0,1,0,1,1)$ & $(0,1,0,1,1)$ & +1 & $(1,0,1,0,0)$ & $(1,0,1,0,0)$ & -1 \\
\hline$(0,0,1,1,1)$ & $(0,0,1,1,1)$ & +1 & $(1,1,0,0,0)$ & $(1,1,0,0,0)$ & -1 \\
\hline
\end{tabular}

Table 4: The Hoede-Bakker index with Standard Procedure

\begin{tabular}{|c|c|c|c|c|c|c|c|c|c|} 
Figure $\rightarrow$ & $1(\mathrm{a})$ & $2(\mathrm{a})$ & $3(\mathrm{a})$ & $3(\mathrm{~b})$ & $3(\mathrm{~d})$ & $3(\mathrm{e})$ & $3(\mathrm{f})$ & $3(\mathrm{~g})$ & $3(\mathrm{~h})$ \\
$H B(k) \downarrow$ & $(\mathrm{b})(\mathrm{c})$ & $2(\mathrm{~b})$ & $3(\mathrm{c})$ & & & & & $3(\mathrm{i})$ & \\
\hline$H B(1)$ & 1 & 1 & $\frac{3}{8}$ & $\frac{3}{4}$ & $\frac{1}{2}$ & $\frac{5}{8}$ & $\frac{1}{2}$ & 1 & $\frac{1}{2}$ \\
\hline$H B(2)$ & 0 & 0 & $\frac{3}{8}$ & 0 & $\frac{1}{2}$ & $\frac{3}{8}$ & 0 & 0 & $\frac{1}{4}$ \\
\hline$H B(3)$ & 0 & 0 & $\frac{3}{8}$ & $\frac{1}{4}$ & 0 & $\frac{3}{8}$ & $\frac{1}{2}$ & 0 & $\frac{1}{2}$ \\
\hline$H B(4)$ & - & 0 & $\frac{3}{8}$ & $\frac{1}{4}$ & $\frac{1}{2}$ & $\frac{1}{8}$ & $\frac{1}{2}$ & 0 & $\frac{1}{4}$ \\
\hline$H B(5)$ & - & - & $\frac{3}{8}$ & $\frac{1}{4}$ & 0 & $\frac{1}{8}$ & 0 & 0 & $\frac{1}{4}$ \\
\hline
\end{tabular}

